$\xi=-1$ 圆

\title{
Design of Wireless Vital Signs Monitoring System for Indoor Monitoring Activity
}

\author{
Philip T. Daely ${ }^{1 *}$, Achmad Rizal' ${ }^{2}$, Sugondo Hadiyoso ${ }^{3}$ \\ ${ }^{1}$ Faculty of Information and Industrial Technology, Institut Teknologi Telkom Surabaya, Surabaya, Indonesia \\ ${ }^{2}$ School of Electrical Engineering, Telkom University, Bandung, Indonesia \\ ${ }^{3}$ School of Applied Science, Telkom University, Bandung, Indonesia \\ *Corresponding author E-mail: philip.daely@ittelkom-sby.ac.id
}

\begin{abstract}
Patient vital signs monitoring is an important activity in medical field, the situation in real medical facility usually does not allow for continuous monitoring. In a medical facility such as hospital or health care center, the patients outnumbered the staffs which makes it difficult to focus on each patient at a time. This problem can decrease chance to efficiently and effectively detecting medical condition. In this paper, we propose a wireless vital signs monitoring system, where multiple patients can be monitored at the same time. The system consists of two parts: Sensor Device to detect and measure patient's vital signs and Monitor Device to present the sensors' measurement result. We use ZigBee protocol for data transmission between devices. We compare our system to previous work to showcase the advantages of our work. We present the performance results related with the ZigBee communication and the display of Graphical User Interface (GUI). The results show that the system is capable of carrying out vital sign monitoring in indoor scenario with interactive measurement display that can help monitor multiple patients simultaneously.
\end{abstract}

Keywords: indoor monitoring, measurement, vital signs, wireless, ZigBee.

\section{Introduction}

Information and communication technologies have penetrated almost all aspects of everyday life, including the medical field. The need for new ideas, methods and techniques that can improve the quality of patient care has led to many studies in the field of biomedical instrumentation for monitoring, therapy, or patien aids. Breakthrough in medicinal technologies such as 3-D printed spinal implants [1, 2], virtual reality for pain relief [3, 4], and surgical robot $[5,6]$ depict many areas to improve the quality of health care.

Vital signs monitoring is one of the important activities carried out in the medical field. This activity can help identifying most of critical health condition if performed regularly and correctly [7, 8]. This activity usually requires continuous attention from doctors or nurses who handle the patients. But there are times when this activity cannot be done continuously for some reasons, such as the patients outnumber the staffs, causing some patients only get attention for small amount of time.

In this paper, we propose a system to monitor patient's vital signs easily. The vital signs being monitored are electrocardiogram (ECG), heart rate, respiratory rate, oxygen saturation $\left(\mathrm{SpO}_{2}\right)$, and temperature. ECG is a signal generated from the electrical activity heart's muscles. Heart rate is measurement of heart beat happening over a period of time, usually in minute. Respiration rate is measurement of how many respirations of human happened over a period of time. $\mathrm{SpO}_{2}$ measures the oxygen saturation in the peripheral capillary, usually in the finger or ear lobe. The body temperature is a measurement of how much heat is generated from the body. We basically make the monitor closer for the staffs to access by making the monitor part and the sensor part separate. The data are transmitted between sensor and monitor parts over wireless network. This would make the medical staffs do not have to be in near proximity with their patient. They can just monitor it at one place, and if something is wrong, then they can rush over towards the patient.

In our proposed design, we incorporate IEEE 802.15.4 transceiver to each devices of wireless network so they can communicate with each other. We use ZigBee protocol to transmit each data because it is suitable for indoor usage and easy to build. ZigBee protocol is also known for its low power feature, which can lower the power consumption of each device and emit less heat to its surrounding. IEEE 802.15.4 standard uses $2.4 \mathrm{GHz}$ of frequency as its medium of transmission, which is part of the Industrial, Scientific, and Medical (ISM) radio bands, allowing it to be used for medical purposes.

Therefore, in this paper, our main contribution includes:

- a novel architecture comprising of wireless centralized vital signed monitoring system with distributed Sensor Devices for each patient

- compact algorithms of Sensor Device and Monitor Device

- performance evaluation of the proposed system, from the sensors, the network, and the monitoring interface side

The remainder of the article is structured as follows. In Section 2, we discuss about related works and include our contribution of this paper showing the difference with existing works. In Section 3 , we comprehensively investigate the proposed system design including communication among devices. Section 4 covers tests results and performance evaluation. Finally, we give our conclusion in Section 5. 
Table 1: Comparison of proposed system with previous works

\begin{tabular}{|c|c|c|}
\hline $\begin{array}{c}\text { Compared } \\
\text { Feature }\end{array}$ & $\begin{array}{c}\text { Proposed } \\
\text { System }\end{array}$ & $\begin{array}{c}\text { Previous } \\
\text { Works }\end{array}$ \\
\hline $\begin{array}{c}\text { Measured } \\
\text { Vital Signs } \\
\text { ECG, heart rate, } \\
\text { respiratory rate, } \mathrm{SpO}_{2}, \\
\text { and } \\
\text { temperature }\end{array}$ & $\begin{array}{c}\text { Heart rate in [9, 10, 11, } \\
\text { 12], respiratory rate in } \\
{[10,11,12], \text { and tem- }} \\
\text { perature in [11, 12] }\end{array}$ \\
\hline $\begin{array}{c}\text { Support multiple patient } \\
\text { monitoring }\end{array}$ & Yes & Yes in [11] \\
\hline $\begin{array}{c}\text { Wireless data } \\
\text { transmission }\end{array}$ & ZigBee & $\begin{array}{c}\text { Bluetooth in [10] } \\
\text { and Wi-Fi in [12] }\end{array}$ \\
\hline $\begin{array}{c}\text { Monitoring } \\
\text { interface }\end{array}$ & PC & $\begin{array}{c}\text { PC in [9, 11, 12] and } \\
\text { smartphone in [10] }\end{array}$ \\
\hline
\end{tabular}

\section{Related Works}

Vital signs are, simply stated, signs of life. They are indications that a human is still alive. Basically, it means that not being able to detect one of them means that the human is probably not in a good condition.

Several works have been done related to vital signs monitoring. The work in [9] proposes a system to measure heart rate from photoplethysmogram (PPG) by using camera. Their proposed system detects the changes of facial color to determine the movement of blood, allowing it to determine the heart rate of the person being measured. The system is also non-invasive as it can measure heart rate from distance.

Other work in [10] proposes a wireless vital signs monitoring system that measure heart rate and respiratory rate in real time. It uses a custom-made microwave-based sensor that first measure the reflection coefficient on patient's chest. The results of measurement are made available through an interface at smartphone, with data transmission over Bluetooth network.

The authors in work [11] use fiber optic-based sensor with capability to measure up to 128 patients simultaneously. By using an integrated Sensor Device, their system can measure heart rate, respiratory rate, and temperature of patient. It is also non-invasive, thus does not affect much of patient's comfort.

Another work in [12] also proposes a system to measure heart rate, respiratory rate, and temperature. The difference is this work make use of Wi-Fi network to transmit measured data to desktop PC for monitoring. The system in this paper shows a good measurement performance when compared to reference medical devices.

In this paper, we propose a system that is different with the previously mentioned works. Our system can monitor multiple patients by employing Sensor Device on each patient. Each Sensor Devices will transmit real time data to central Monitor Device over ZigBee network. Our system is also designed to measure five vital signs: ECG, heart rate, respiratory rate, $\mathrm{SpO}_{2}$, and temperature. The comparison is shown in Table 1.

\section{Proposed System Design}

The proposed system design comprises of two parts: Sensor Device and Monitor Device. The Sensor Device is designed for detecting measuring vital signs on patient's body. Whereas the Monitor Device is designed to show the measurement results for the medical staffs. The block diagram of our proposed system is shown in Fig. 1.

Our proposed system utilizes ZigBee network as medium of communication between devices. ZigBee is one of communication protocols for Low-Rate Wireless Personal Area Network (LRWPAN). It is developed based on IEEE 802.15.4 standard by ZigBee Alliance. ZigBee protocol functions over three different frequency: $868 \mathrm{MHz}, 915 \mathrm{MHz}$, and $2.4 \mathrm{GHz}$. It can transmit up to $40 \mathrm{~m}$ indoor and $100 \mathrm{~m}$ outdoor [13].

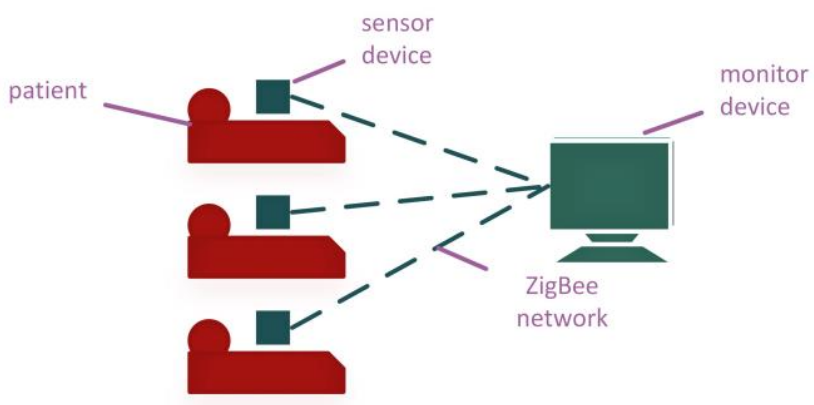

Fig. 1: The block diagram of proposed system

There are three types of ZigBee devices: ZigBee Coordinator (ZC), ZigBee Router (ZR), and ZigBee End-Device (ZED). ZC is the coordinator of the network. It is the device that builds the network infrastructure and assigns identification number to every joining device. ZR is device in ZigBee network with capability of extending ZigBee network range and record every route from and to every neighboring device. ZED is the last type of device that usually used for Sensor Devices. It is capable of lowering its energy usage to save energy by periodically turning itself off when not active. In our system, we setup the ZigBee transceiver at Monitor Device as ZC and ZigBee transceiver at each Sensor Device as ZED.

\subsection{Design of Sensor Device}

The Sensor Device consists of multiple sensors to measure the vital signs of patient. This device is designed with microcontroller as its main processor. The microcontroller will process all data from every sensor and then send it to the Monitor Device through ZigBee transceiver. The block diagram of Sensor Device is shown in Fig. 2.

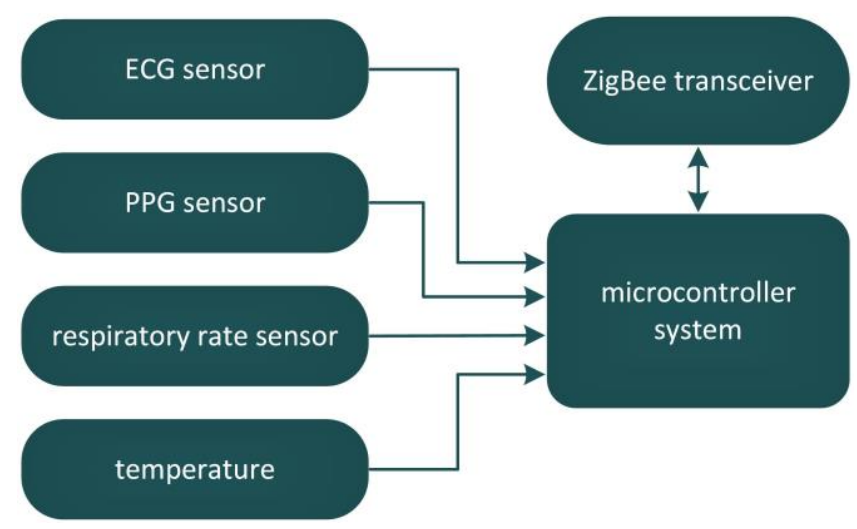

Fig. 2: The block diagram of Sensor Device.

For our evaluation purpose, we build the prototype of Sensor Devices, with microcontroller ATMega328 as the main processor. The microcontroller is powered with $5 \mathrm{~V}$ dc voltage, with 6channel 10-bit analog-to-digital converter (ADC), 23 general purpose digital I/O lines, three timer/counter modules, a Universal Serial Asynchronous Receiver/Transceiver (USART) module, and 32 KB In-System Programming (ISP) memory [14]. We use electrodes connected to microcontroller through ADC channel to detect ECG signal from the patient body. For the heart rate and $\mathrm{SpO}_{2}$ measurement, we use CMS50DL Pulse Oximeter connected to microcontroller through digital port. Respiration rate is measured by using thermocouple sensor, placed on the nose of patient, and connected to microcontroller through ADC channel. The patient's body temperature is measured by using thermistor connected to microcontroller's ADC channel. For data transmission, we use 
XBee S2, which is connected with microcontroller through USART module.

The brief process of vital signs measurement by Sensor Device is as follows. At first, the microcontroller will read the output of each sensor and convert it to a meaningful result of measurement. After that, it will arrange the results into a packet according to a designated format. Finally, microcontroller will send the packet to ZigBee transceiver to be sent to Monitor Device. The flowchart of vital signs measurement is shown in Fig. 3.

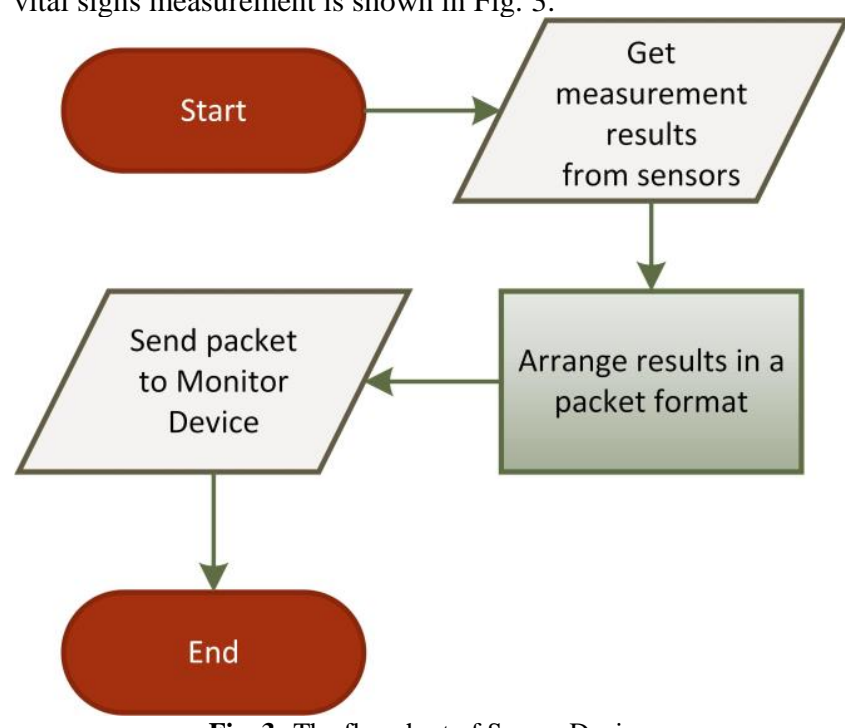

Fig. 3: The flowchart of Sensor Device.

\subsection{Design of Monitor Device}

The design of Monitor Device is made with desktop PC as its main part. We connect the ZC to desktop PC through Universal Serial Bus (USB) port. We also design the Graphical User Interface (GUI) in a laptop PC using Microsoft Visual Studio. The PC we use for this work is a 64-bit Acer Nitro 5 laptop PC, with 2.5 $\mathrm{GHz}$ Intel Core i5-7300HQ central processor unit (CPU), Nvidia GeForce GTX1050 graphics processing unit (GPU), and 8 GB random-access memory (RAM). The PC uses Windows 10 as its operating system. The diagram block of Monitor Device is shown in Fig. 4.

The process of retrieving and displaying vital signs measurement from Sensor Device by Monitoring Device is as follows. The GUI will first read the feed from ZigBee transceiver connected to PC through USB port. When it gets the packet from Sensor Device, it will parse each measurement results from the packet. The GUI then will display ECG and airflow in real-time line chart and heart rate, respiratory rate, $\mathrm{SpO}_{2}$, and temperature in real-time numerical form. The flowchart of vital signs measurement is shown in Fig. 5.

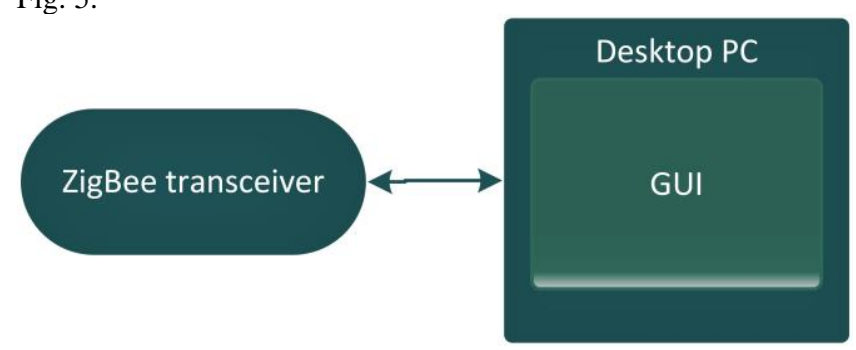

Fig. 4: The block diagram of Monitor Device.

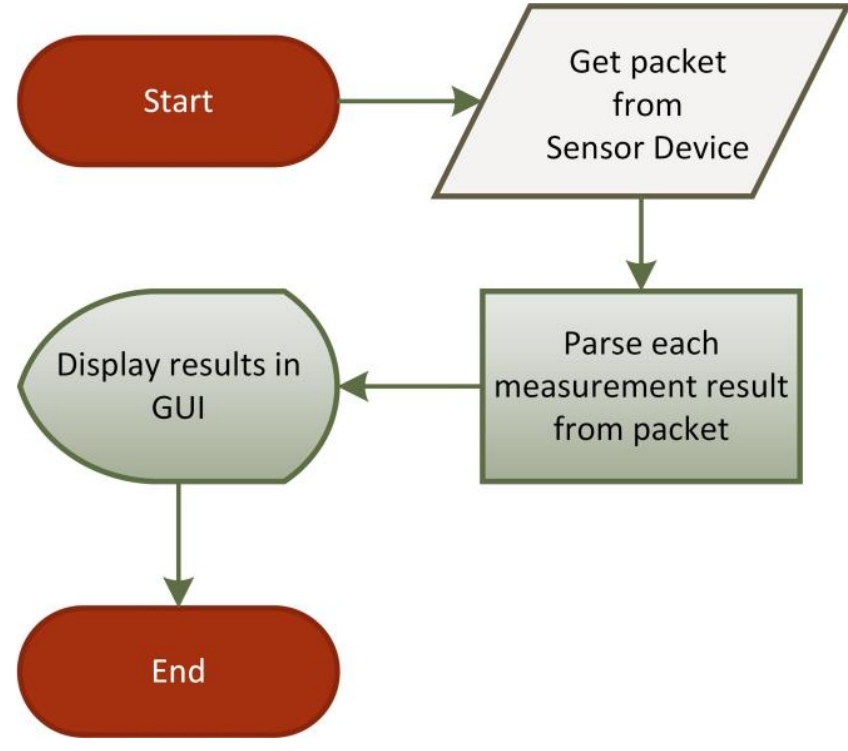

Fig. 5: The flowchart of Monitor Device.

\section{Performance Evaluation}

In this section, we present our evaluation of the proposed system. We do not evaluate the sensors' performance because they are mass fabricated and available in the market. The sensors are also replaceable by similar products that can measure the required vital signs. Our evaluation emphasizes more on the ZigBee network and GUI of proposed system.

\subsection{ZigBee Network Evaluation}

ZigBee network is one of vital parts in our proposed system. The performance of the system is depending on how well every device communicate to each other. One of indicator that can be measured to determine its performance is the Received Signal Strength (RSS). RSS is the measurement of power from received signal in $\mathrm{dBm}$. The RSS will be stronger when the distance between two wireless devices is shorter.

In our evaluation, we considered two ZigBee transceivers to act as Sensor Device and Monitor Device. We evaluate the RSS from Monitor Device side with respect to transmission distance with Sensor Device. For our prototype, we use XBee S2 as ZigBee transceiver. The evaluation is done at the ground floor of Institut Teknologi Telkom Surabaya, as shown in Fig. 6. The green circle represents the position of Monitor Device and the red circle represent the position of Sensor Device. Both devices are in Line-ofSight (LOS) environment inside a building. The distance between devices is varied from $1 \mathrm{~m}$ to $40 \mathrm{~m}$ with interval of $1 \mathrm{~m}$.

The evaluation results of RSS measurement between two of our devices is shown in Fig. 7. The blue line in Fig. 7 shows that it can still receive signal well until $30 \mathrm{~m}$. The RSS does not fall significantly until $30 \mathrm{~m}$. We can also see that the Packet Delivery Rate, showed by the orange line in Fig. 7, is stable with distance under $30 \mathrm{~m}$, but it starts to fluctuate slightly larger when the distance is extended over $30 \mathrm{~m}$.

\subsection{GUI Evaluation}

The GUI shown in Fig. 8 is designed using C\# language in Microsoft Visual Studio. Beside the vital signs measurement, the GUI also equipped with two buttons, each is for connecting and disconnecting GUI from the USB port. The data stream of USB is also displayed to help ensuring the data is coming from the real Sensor Device and in a correct format. The date and time of the 


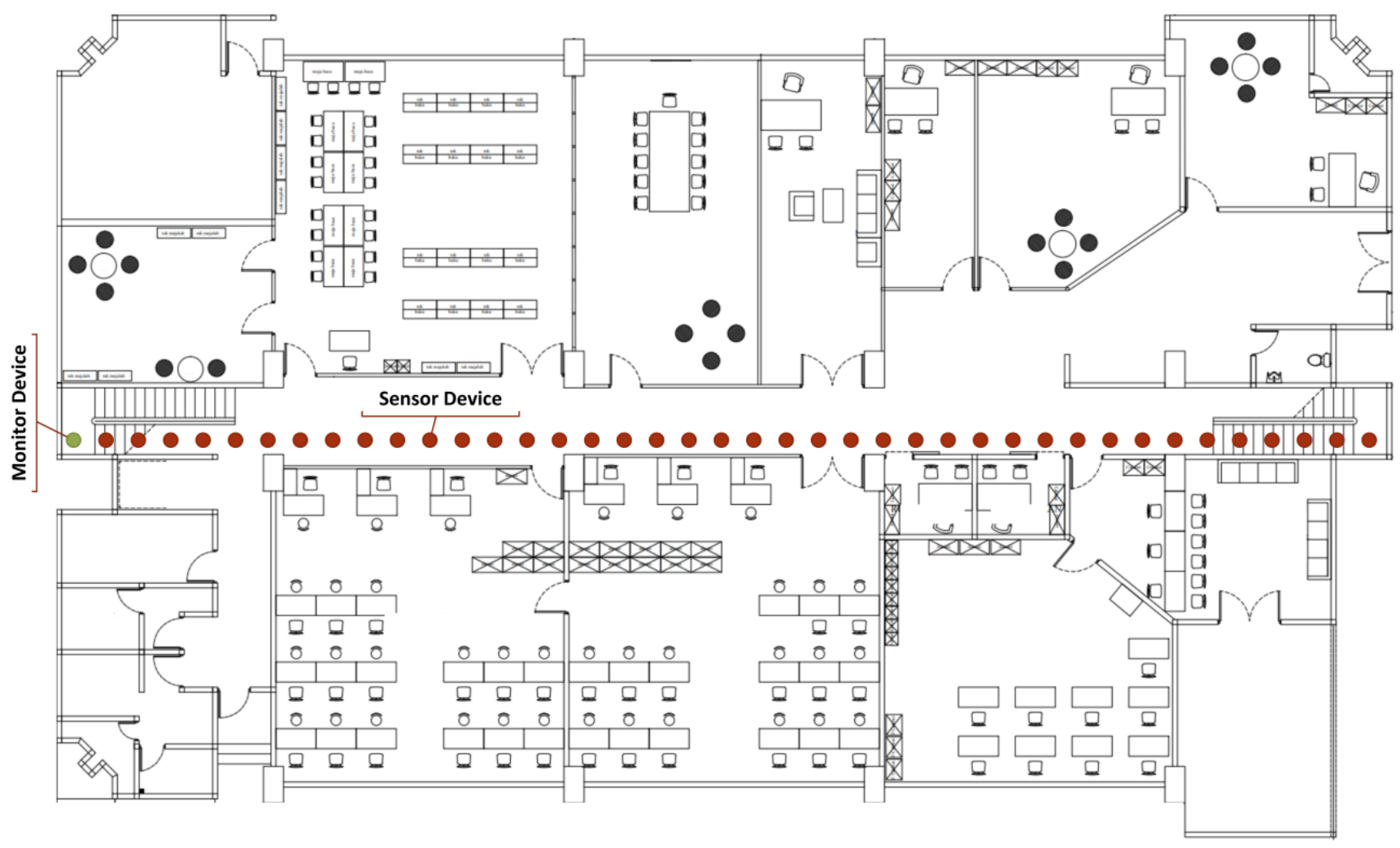

Fig. 6: The flowchart of Monitor Device.

day of measurement are also shown to show that the measurement is in real time and always up-to-date with the current measurement.

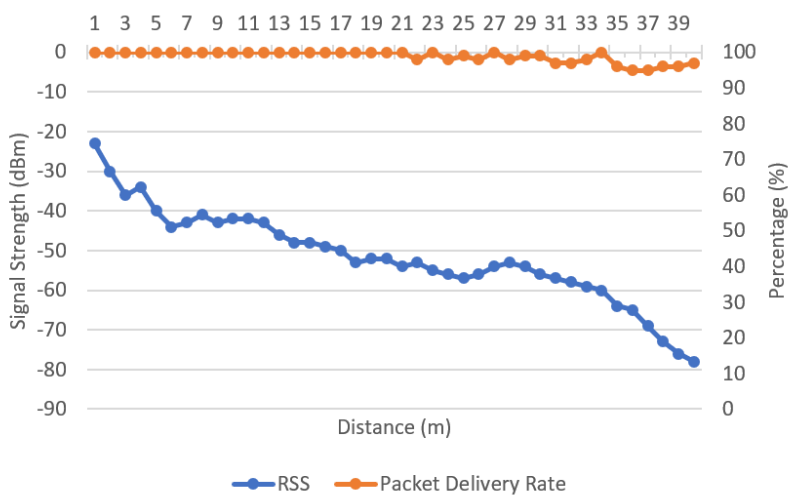

Fig. 7: The result of transmission performance measurement over ZigBee.

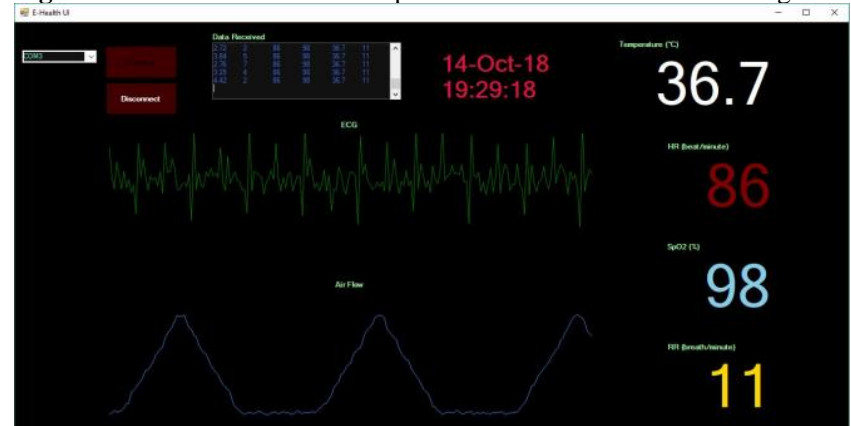

Fig. 8: The display of GUI when receiving measurement results from Sensor Device.

The result shows a good display, showing the real time graphic of ECG and air flow of respiration, and also numerical measurements of temperature, heart rate, $\mathrm{SpO}_{2}$, and respiratory rate. The GUI still includes the noise originated from the Sensor Device and thus need additional digital filter to mitigate the noise and output a decent graph for ECG and airflow.

\section{Conclusion and Future Works}

This paper has discussed a compact design of wireless vital signs monitoring system. From the performance evaluation, we can see that ZigBee is fit for indoor medical monitoring. With benefits of ZigBee protocol, we can easily measure multiple patients on the same time. The evaluation points out that data transmission can still be done with good quality until $30 \mathrm{~m}$. This distance is considered to be relatively suitable for indoor usage. The designed GUI also show a good display of vital signs measurement. Further modification is necessary to increase the display quality, but overall, it can receive data well from the Sensor Device and give an intuitive measurement result.

Future works include designing a better GUI, which can be run on any computing platform, such as computer and smartphone. The system also needs to be connected to internet, so people from far places can use the system as well. Recording patient's data history in database is also necessary for continuous monitoring over a long time.

\section{Acknowledgement}

This work is sponsored by Institut Teknologi Telkom Surabaya. We would also like to give our gratitude towards Biomedical Signal Processing and Instrumentation Laboratory of Telkom University for providing aid in designing our prototype.

\section{References}

[1] N. Xu, F. Wei, X. Liu, et al., "Reconstruction of the Upper Cervical Spine Using a Personalized 3D-Printed Vertebral Body in an Adolescent with Ewing Sarcoma," Spine, vol. 41, no. 1, pp. E50-E54, Jan 2016, available online: https://www.ncbi.nlm.nih.gov/pubmed/ 26335676 
[2] W. J. Choy, R. J. Mobbs, B. Wilcox, S. Phan, K. Phan, and C. E. S. III, "Reconstruction of Thoracic Spine Using a Personalized 3DPrinted Vertebral Body in Adolescent with T9 Primary Bone Tumor," World Neurosurgery, vol. 105, pp. 1032.e13-1032.e17, Sep 2017, available online: https://www.ncbi.nlm.nih.gov/pubmed/ 28578109

[3] S. M. Alshatrat, R. Alotaibi, M. Sirois, and Z. Malkawi, "The Use of Immersive Virtual Reality for Pain Control During Periodontal Scaling and Root Planing Procedures in Dental Hygiene Clinic," International Journal of Dental Hygiene, vol. 0, no. 0, Sep 2018 , available online: https://www.ncbi.nlm.nih.gov/pubmed/30216688

[4] E. Ambron, A. Miller, K. J. Kuchenbecker, L. J. Buxbaum, and H. B. Coslett, "Immersive Low-Cost Virtual Reality Treatment for Phantom Limb Pain: Evidence from Two Cases," Frontiers in Neurology, vol. 9, p. 67, Feb 2018, available online: https://www.ncbi. nlm.nih.gov/pubmed/29515513

[5] M. J. Kim, S. C. Park, J. W. Park, et al., "Robot-assisted Versus Laparoscopic Surgery for Rectal Cancer: A Phase II Open Label Prospective Randomized Controlled Trial," Annals of Surgery, vol. 267, no. 2, pp. 243-251, Feb 2018, available online: https://www. ncbi.nlm. nih.gov/pubmed/28549014

[6] H. Ohtani, K. Maeda, S. Nomura, et al., "Meta-analysis of Robotassisted Versus Laparoscopic Surgery for Rectal Cancer," In Vivo, vol. 32, no. 3, p. 611-623, May 2018, available online: https:// www.ncbi.nlm.nih.gov/pmc/articles/PMC6000779

[7] G. A. Harrison, T. C.Jacques, G. Kilborn, and M.-L. McLaws, "The Prevalence of Recordings of the Signs of Critical Conditions and Emergency Responses in Hospital Wards - the SOCCER Study," Resuscitation, vol. 65, no. 2, pp. 149-157, May 2005, available online: https://www.ncbi.nlm.nih.gov/pubmed/15866394

[8] K. Fagan, A. Sabel, P. S. Mehler, and T. D. MacKenzie, "Vital Sign Abnormalities, Rapid Response, and Adverse Outcomes in Hospitalized Patients," American Journal of Medical Quality, vol. 27, no. 6, pp. 480-486, Nov 2012, available online: https://www.ncbi.nlm. nih.gov/pubmed/22378957

[9] M. Kumar, A. Veeraraghavan, and A. Sabharwal, "DistancePPG: Robust Non-contact Vital Signs Monitoring Using a Camera," Biomedical Optimal Express, vol. 6, no. 5, pp. 1565-1588, May 2015, available online: https://www.ncbi.nlm.nih.gov/pubmed/26137365

[10] D. Bibb, R. R. G. Perron, G. C. Huang, and M. F. Iskander, "Development of a Wireless Monitoring System for Microwave-Based Comprehensive Vital Sign Measurement," IEEE Antennas and Wireless Propagation Letters, vol. 15, pp. 1249-1252, May 2016, available online: https://ieeexplore.ieee.org/document/7347348

[11] M. Fajkus, J. Nedoma, R. Martinek, V. Vasinek, H. Nazeran, and P. Siska, "A Non-Invasive Multichannel Hybrid Fiber-Optic Sensor System for Vital Sign Monitoring," Sensors, vol. 17, no. 1, Jan 2017, available online: https://www.ncbi.nlm.nih.gov/pmc/articles/ PMC5298684

[12] G. Sun, T. Matsui, Y. Watai, S. Kim, T. Kirimoto, S. Suzuki, and Y. Hakozaki, "Vital-SCOPE: Design and Evaluation of a Smart Vital Sign Monitor for Simultaneous Measurement of Pulse Rate, Respiratory Rate, and Body Temperature for Patient Monitoring," Journal of Sensors, vol. 2018, Feb 2018, available online: https://www.hindawi.com/journals/js/2018/4371872

[13] S. Farahani, "Chapter 1 - ZigBee Basics," in ZigBee Wireless Networks and Transceivers, S. Farahani, Ed. Burlington: Newnes, 2008, pp. 1-24, available online: https://www.sciencedirect.com/science/ article/pii/B9780750683937000017?via\%3Dihub

[14] ATmega48A/PA/88A/PA/168A/PA/328/P megaAVR® Data Sheet, Microchip, 1 2018, available online: https://www.microchip.com/ wwwproducts/en/ATmega328 